\title{
On the Importance of Image Gating for the Assay of Left Ventricular Mechanical Dyssynchrony Using SPECT
}

\author{
Daniel R. Ludwig, Mati Friehling, David Schwartzman, Samir Saba, William P. Follansbee, and Prem Soman \\ Heart and Vascular Institute, University of Pittsburgh, Pittsburgh, Pennsylvania
}

The potential of SPECT for quantifying left ventricular mechanical dyssynchrony is increasingly appreciated. We sought to examine the incidence and impact of image gating errors on this quantification and to test a possible solution for affected studies. Methods: First, to establish whether and how gating error alone could affect the measurement of dyssynchrony, we performed a prospective study in which patients with pacemakers were studied twice: during normal rhythm without gating error and with gating error caused by pacemaker-induced dysrhythmia. Second, to understand the pattern and magnitude of gating error during our typical imaging practice, we retrospectively examined studies from a separate cohort of 64 patients who were referred for dyssynchrony evaluation. Third, to understand whether studies with gating error could be repaired for the purpose of quantifying dyssynchrony, we tested a correction algorithm on the pacemaker-induced dysrhythmia image set to see whether it repaired this set so as to approximate the patients' normal rhythm image data. We subsequently applied this algorithm to the 64-patient cohort. Results: Pacemaker-induced gating error caused a spurious decrease in dyssynchrony magnitude. Among the 64-patient cohort, similar gating errors were common, and an inverse exponential relationship between gating-error magnitude and dyssynchrony magnitude was observed. The correction algorithm accurately repaired the pacemaker-induced dysrhythmia image set; when it was applied to the 64-patient cohort, the magnitude of the postcorrection increase in dyssynchrony magnitude was proportional to the magnitude of the gating error. Conclusion: Gating errors cause a spurious reduction in SPECT assay of dyssynchrony magnitude. In our standard imaging practice, gating errors were common. Post hoc correction appears to be feasible.

Key Words: SPECT; dyssynchrony; left ventricle; heart failure; pacing; resynchronization

J Nucl Med 2012; 53:1892-1896

DOI: 10.2967/jnumed.112.106344

\section{A} ure and left ventricular (LV) dyssynchrony using cardiac

\footnotetext{
Received Mar. 25, 2012; revision accepted Jul. 9, 2012.

For correspondence or reprints contact: Prem Soman, A-429 Scaife Hall, 200 Lothrop St., UPMC Presbyterian, Pittsburgh, PA 15213-2582.

E-mail: somanp@upmc.edu

Published online Nov. 9, 2012.

COPYRIGHT @ 2012 by the Society of Nuclear Medicine and Molecular Imaging, Inc.
}

resynchronization therapy fails in more than $30 \%$ of patients, a serious limitation for this invasive and expensive treatment (1). The utility of phase analysis of SPECT for assessing LV synchrony and thereby improving patient selection for cardiac resynchronization therapy is increasingly apparent (24). In assessing the robustness of this technique, an issue that must be addressed is image gating. Our experience has suggested that gating errors may introduce artifacts that affect phase analysis results. In the present study, we sought to confirm this notion, examine its mechanism, quantify its incidence and impact, and test a method for post hoc correction of affected studies.

\section{MATERIALS AND METHODS}

Studies involving patients were sanctioned by the Institutional Review Board of the University of Pittsburgh Medical Center.

\section{Whether and How Gating Error Affects SPECT Phase Analysis}

SPECT images were acquired on a dual-head $\gamma$-scintillation camera (Philips Inc.) after the administration of high-dose (1,110 MBq) ${ }^{99 \mathrm{~m}} \mathrm{Tc}$-sestamibi using standard myocardial perfusion imaging parameters (20\% acceptance window around a $140-\mathrm{keV}$ energy peak; $180^{\circ}$ orbit; and 32 steps, with $25 \mathrm{~s}$ of data acquisition per step). Gated tomograms were acquired with 16 frames ( 64 projections per frame) per RR interval, with a beat-acceptance window of between $40 \%(80 \%-120 \%$ RR) and $100 \%(50 \%-150 \%$ RR). The longer beat-acceptance windows were used in patients with atrial fibrillation or frequent ectopy. Iterative reconstruction was performed with a filtered backprojection start and a maximum-likelihood expectation maximization algorithm (12 iterations), using a Butterworth filter with a cutoff frequency of 0.66 and an order of 5 .

Patients referred for SPECT as part of their standard clinical care were screened for the following criteria: they 1 . were in normal sinus rhythm; 2. had an implanted defibrillator or pacemaker system, which included a right atrial lead; 3. had intact atrioventricular conduction; and 4. had diminished LV ejection fraction. Five patients were enrolled (4 men, 1 woman; 4 with ischemic etiology; ejection fraction was $30 \% \pm 14 \%)$. Each enrolled patient underwent SPECT during the following 2 conditions: 1. normal sinus rhythm without asynchronous atrial pacing and 2. normal sinus rhythm with asynchronous atrial pacing. In the latter condition, pacing would occur randomly at a low rate (30/ min). When pacing stimuli were able to capture the atrium and find the atrioventricular node nonrefractory, they imparted an irregularity to the ventricular rhythm that did not alter the intraventricular electromechanical activation sequence. As expected, 


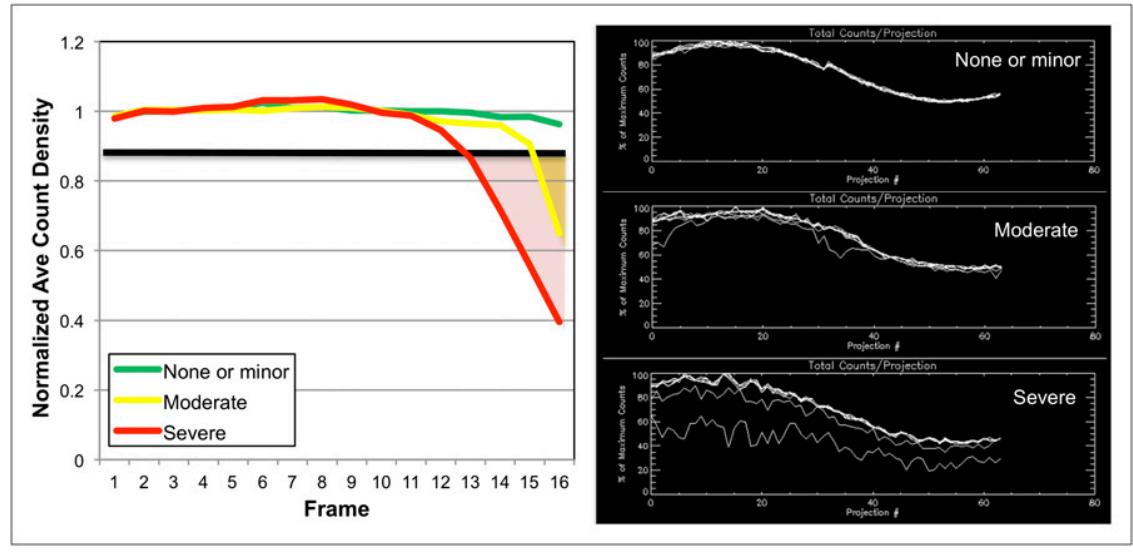

FIGURE 1. Quantitative assessment of gating-error magnitude. (Left) Normalized count density is shown across each frame of cardiac cycle for 3 example patients, demonstrating no or minor gating error, moderate gating error, and severe gating error. Gating-error magnitude was quantified by percentage of area that fell below $90 \%$ line but above intensity-time curve (shaded; $0 \%$ for none or minor, $0.9 \%$ for moderate, and $5.9 \%$ for severe). (Right) Count projection curves for same 3 patients using Emory Cardiac Toolbox, which reported gating error as none or minor (green), moderate (yellow), and severe (red). Ave = average.

between conditions we observed no significant difference in RR interval (condition 1, $716 \pm 164 \mathrm{~ms}$; condition 2, $671 \pm 122 \mathrm{~ms}$; $P=$ not significant) but a significant difference in RR variability (condition 1, 5.2\% $\pm 3.6 \%$; condition $2,29 \% \pm 10 \% ; P<0.05$ ).

Images obtained during each condition were subjected to assessment of LV mechanical synchrony using the Emory Cardiac Toolbox (Synctool; Syntermed Inc.), which uses a Fourier-based approach as described previously (5). Because the count density of a myocardial region during the cardiac cycle is linearly related to myocardial thickness as a result of the partial-volume effect (6), the time-activity curve represents its radial thickening profile. A fast Fourier transform is performed on the 16-frame time-activity curves for each of over 600 separate myocardial regions. The temporal onset of mechanical contraction during the cardiac cycle of each region is determined using the phase of the first Fourier harmonic. Synchrony is quantified by the SD of the phase of mechanical activation (PSD). A higher value for this parameter denotes less normal LV contraction synchrony; on the basis of previously published data, we used a PSD value of more than $24.4^{\circ}$ for men and more than $22.2^{\circ}$ for women as definitions of dyssynchrony $(2,5)$.

\section{Incidence, Magnitude, and Impact of Gating Error in Typical Imaging Practice}

We retrospectively examined the data from each of 64 patients (51 men, 13 women; mean age $\pm \mathrm{SD}, 66 \pm 11 \mathrm{y}$; ischemic etiology in 43) who had most recently undergone imaging in our laboratory as part of an ongoing clinical trial and met standard criteria for cardiac resynchronization therapy: established syndrome of congestive heart failure (New York Heart Association classification, $2.8 \pm 0.6$ ), electrocardiographic QRS duration more than $120 \mathrm{~ms}(160 \pm 30 \mathrm{~ms})$, and LV ejection fraction less than $35 \%(27 \% \pm 6 \%)$. Of these, $11(17 \%)$ had atrial fibrillation and 18 $(28 \%)$ had significant atrial or ventricular ectopy.

In assessing for gating error, count drop-off was identified by averaging the image intensity in the entire reconstructed short-axis stack for each of the 16 frames and normalizing the intensity to that of the average of the first 5. Error magnitude was quantified by the percentage area that fell below the $90 \%$ line but above the intensity-time curve: less than $0.50 \%$ (none or minor), $0.51 \%$ $1.0 \%$ (moderate), or more than $1.01 \%$ (severe) (Fig. 1). This method was compared with the Emory Cardiac Toolbox qualitycontrol indicator, which uses a proprietary algorithm to assign gating-error magnitude on the basis of discordance, relative to average counts among all projections comprising each frame before reconstruction, between the count-projection curves for alter- nate (odd) frames: 1. none or minor: no count drop-off in any frame; 2 . moderate: count drop-off in 1 frame; and 3. severe: count drop-off in multiple frames.

\section{Post Hoc Correction of Affected Studies}

With a single exception, any frames in the reconstructed short axis whose intensity was less than $90 \%$ of the first 5 frames were empirically scaled up to the average intensity of the first 5 frames. The exception to this was for severely affected frames (normalized intensity $<10 \%$ ), which were removed completely and replaced with a replicate of the first frame to avoid scaling up images with a compromised signal-to-noise ratio.

\section{Statistical Methods}

Data are represented as mean $\pm \mathrm{SD}$, unless otherwise stated. Continuous data were compared using the Student $t$ test in a paired or unpaired manner where appropriate. Correlations were performed using Pearson product-moment analysis. For each test, a $P$ value of less than 0.05 was considered significant.

\section{RESULTS}

\section{Whether and How Gating Error Affects SPECT Phase Analysis}

Among the 5 patients studied, relative to sinus rhythm (PSD, $49.9^{\circ} \pm 21.4^{\circ}$ ), a significant decrease in PSD was observed during asynchronous atrial pacing $\left(18.7^{\circ} \pm 8.2^{\circ}\right.$; $P<0.05)$. These artifacts were typical of gating artifacts in our experience, which are seen as flickering or flashing in the reconstructed short-axis cine loop and are the result of count drop-off in the latter frames of the cardiac cycle. They occur because of the inclusion of beats that have a shorter RR interval than that at baseline but still fall within a prespecified acceptance window.

TABLE 1

Simulated Gating Error Results in Significant Drop-Off in PSD

\begin{tabular}{lrrc}
\hline Severity & Gating error & Change in PSD & $P$ vs. baseline \\
\hline 1 & $0.1 \% \pm 0.1 \%$ & $-11.8 \% \pm 7.7 \%$ & 0.01 \\
2 & $0.8 \% \pm 0.2 \%$ & $-23.5 \% \pm 9.4 \%$ & 0.001 \\
3 & $2.4 \% \pm 0.4 \%$ & $-41.0 \% \pm 12.2 \%$ & $<0.001$ \\
4 & $6.3 \% \pm 0.3 \%$ & $-57.2 \% \pm 11.8 \%$ & $<0.001$ \\
5 & $12.9 \% \pm 0.3 \%$ & $-69.2 \% \pm 9.2 \%$ & $<0.001$ \\
\hline
\end{tabular}




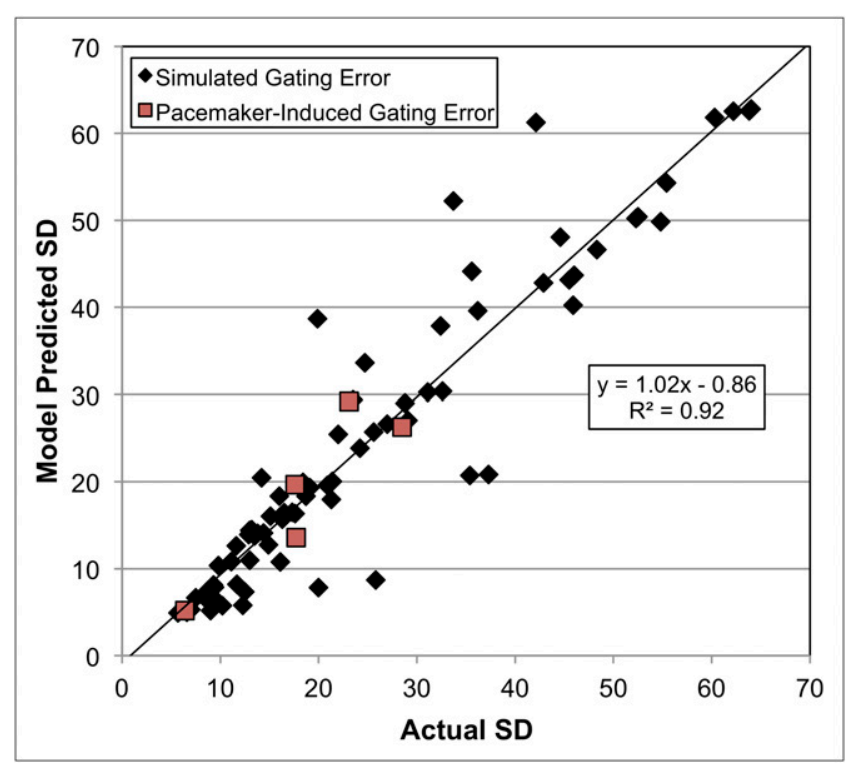

FIGURE 2. Modeling of PSD in presence of gating error. Multiparameter model $\left(P S D=[0.40] \times[\text { true PSD }]^{1.2} \times \mathrm{e}^{(- \text {severity/4.6) }}+\right.$ 4.3) describes drop in PSD occurring with both simulated and pacing-induced gating error. Data from simulation studies are pooled from 12 patients, using each of 5 simulated severities of gating error per patient.

To better quantify the relationship between gating error and PSD, we simulated gating error using the 12 patients from the 64-patient cohort whose studies demonstrated perfect gating. In each simulation study, we artificially scaled down the counts in the last 4 (of 16) gates of the cardiac cycle. The scaling was performed using a range of severities but maintained a drop-off ratio of 0.9:0.75:0.55:0.3 to emulate

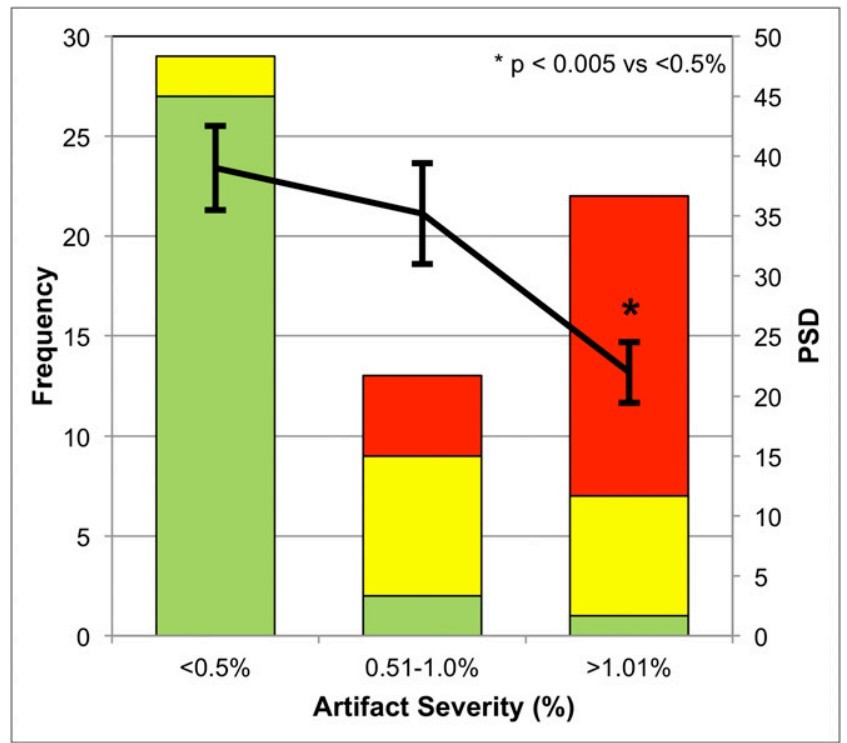

FIGURE 3. Prevalence and influence of gating error on PSD in our 64-patient cohort. Bars represent frequency of gating error at each level of severity; each bar is stratified by Emory Cardiac Toolbox's report of gating error (green, none or minor; yellow, moderate; and red, severe). Overlain is PSD for each group (mean $\pm \mathrm{SE}$ ).
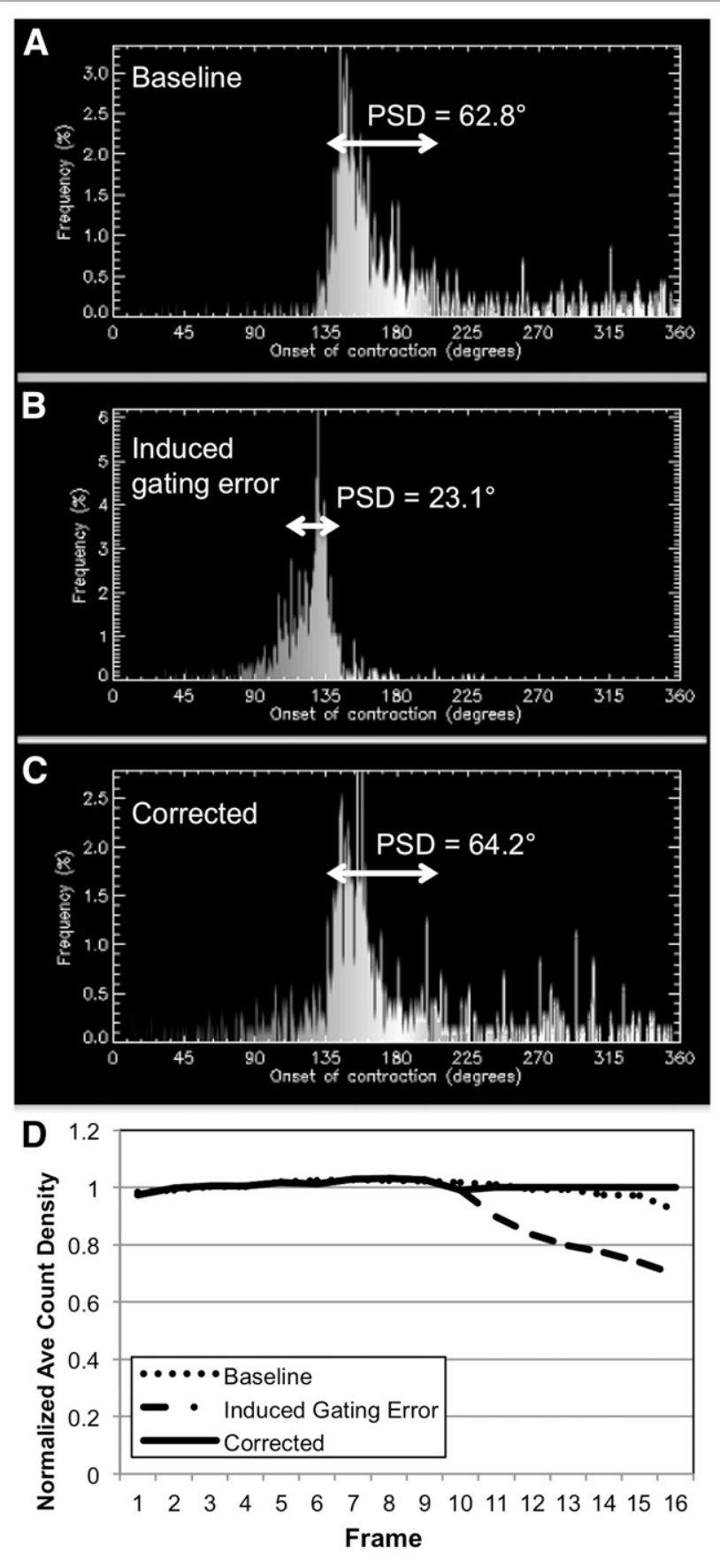

FIGURE 4. Post hoc correction of gating error in 1 patient. (A-C) Phase histograms from normal sinus rhythm study $(A)$, pacemakerinduced gating-error study before application of correction algorithm (B), and pacemaker-induced gating-error study after application of correction algorithm (C). Each histogram shows percentage of myocardium ( $y$-axis) contracting at each phase of cardiac cycle ( $x$-axis). (D) Count drop-off pattern occurring with pacing-induced gating-error study; correction raises counts in frames with drop-off to values comparable to baseline.

patterns of gating error that are common in our experience. We observed an exponential decay in PSD as gating-error severity increased, with an eventual plateau (Table 1). Using these data, we developed a multiparameter model to 
describe PSD as a function of gating-error severity (\%) and true PSD (measured using the perfectly gated study):

$$
\operatorname{PSD}=(0.40) \times(\text { true PSD })^{1.2} \times \mathrm{e}^{(- \text {severity/4.6)}}+4.3 .
$$

Data obtained from the 5 patients in whom suboptimal gating was induced by pacing fit this relationship well (Fig. 2).

\section{Incidence, Magnitude, and Impact of Gating Error in Typical Imaging Practice}

Among the 64-patient cohort, gating errors were none or minor in $29(45 \%)$, moderate in $13(20 \%)$, and severe in $22(35 \%)$ individuals within the cohort. This result correlated reasonably well with the Emory Cardiac Toolbox quality-control indicator. As anticipated from our simulation studies, PSD decreased in proportion to the severity of the gating error (Fig. 3).

\section{Post Hoc Correction of Affected Studies}

We corrected affected studies by scaling up the intensity of frames we identified as having count drop-off. We first tested the correction algorithm in the 5 patients detailed above who had undergone both a sinus rhythm study without asynchronous atrial pacing (study 1) and a sinus rhythm study with asynchronous atrial pacing (study 2). The first study was used to judge the accuracy of the correction of the second study. In each patient, the corrected PSD value from study 2 was nearly identical to the PSD value from study 1 (Fig. 4; Table 2).

Next, we applied the correction algorithm to images from the 35 members of the 64-patient cohort whom we had previously identified as having moderate or severe errors. In this group, the postcorrection PSD $\left(45.1^{\circ} \pm 20.1^{\circ}\right)$ was significantly greater than the precorrection $\operatorname{PSD}\left(26.9^{\circ} \pm 14.4^{\circ}\right.$, $P<0.001)$; in applying these changes, the prevalence of dyssynchrony rose from $54 \%$ before correction to $80 \%$ after correction. The application of the correction algorithm to the image data of 29 patients with no or minor gating errors still also resulted in a postcorrection PSD $(44.7 \pm 21.7)$ that was significantly larger than the precorrection PSD, albeit minimally $(39.0 \pm 19.0, P<0.05)$; in applying these changes, the prevalence of dyssynchrony rose from $72 \%$ before correction to $79 \%$ after correction. Similarly, among the 30 patients who were identified as having no or minor gating errors using the Emory Cardiac Toolbox, 3 (10\%) would have been reclassified as dyssynchronous.

\section{DISCUSSION}

Herein, we substantiate that gating errors can affect SPECT analysis of LV synchrony, and we demonstrate the incidence, magnitude, and relevance of this phenomenon in our typical imaging practice. Although gating errors are usually attributable to disturbances of cardiac rhythm such as atrial fibrillation or frequent ectopic beats, in our experience the baseline electrocardiogram has limited value for predicting gating error.

Gating error is well known to affect the veracity of other SPECT-derived functional parameters, including wall thickening $(7,8)$. We are not aware of a prior report focusing on the impact of gating errors on the assessment of LV mechanical synchrony. Previous reports of the use of SPECT for this purpose either did not address the gating issue $(3,4,9-13)$ or did not include patients for whom a gating error was thought to be present (albeit not quantified) (2). Our data would suggest that all studies should be scrutinized for gating error and that even those with minor errors should be repaired.

The mechanism by which gating error yields a spurious decrease in PSD is based on count drop-off in frames late in the cardiac cycle, which likely alters the fit of the first sinusoidal harmonic to this perceived trough at the end of the cardiac cycle, the magnitude of which is proportional to that of the drop-off. As is suggested by our model, this effect is magnified at longer PSDs, accentuating its impact on patients with diminished ejection fraction, who are typically referred for the synchrony assay. Our investigation of this effect is applicable specifically to synchrony assessed using a count-based approach, and its applicability to a geometric approach (14) remains to be determined.

The best approach to avoiding gating-error artifacts is an image acquisition workflow in which gating is a particular focus, for example, using a narrow beat-acceptance window. However, this would be a significant departure from current practice and would likely extend the study duration or require retrospective (list-mode) gating (15), a technique not widely available on standard $\gamma$-camera equipment. More important, given that dysrhythmia is common among referred patients, many studies would still have gating errors. Clearly, if SPECT analysis of LV mechanical synchrony is going to be robust and widely applicable, a correction algorithm will be mandatory. Our experience with

TABLE 2

Correction of Pacing-Induced Gating Error Restores PSD to That of Baseline Study

\begin{tabular}{|c|c|c|c|c|}
\hline Patient no. & Baseline PSD & Induced gating error & Induced gating error PSD & Corrected PSD \\
\hline 1 & $70.3^{\circ}$ & $5.0 \%$ & $28.5^{\circ}$ & $64.8^{\circ}$ \\
\hline 2 & $52.2^{\circ}$ & $5.0 \%$ & $17.6^{\circ}$ & $54.9^{\circ}$ \\
\hline 3 & $14.7^{\circ}$ & $11.2 \%$ & $6.4^{\circ}$ & $18.0^{\circ}$ \\
\hline 4 & $49.3^{\circ}$ & $7.1 \%$ & $17.7^{\circ}$ & $59.3^{\circ}$ \\
\hline 5 & $62.8^{\circ}$ & $3.8 \%$ & $23.1^{\circ}$ & $64.2^{\circ}$ \\
\hline Average & $49.9^{\circ} \pm 21.4^{\circ}$ & $6.4 \% \pm 2.9 \%$ & $18.7^{\circ} \pm 8.2^{\circ}$ & $52.2^{\circ} \pm 19.6^{\circ}$ \\
\hline$P$ vs. baseline & & & 0.007 & 0.64 \\
\hline
\end{tabular}


one such algorithm is promising but requires prospective confirmation in a larger cohort. The application of this algorithm to prereconstruction image data might be even more accurate but would be more difficult to implement in the current clinical workflow. Other algorithms might also be effective, for example, fitting a sinusoid only to frames with preserved counts or using a mathematic model to calculate the true PSD from the measured PSD and gatingerror severity.

\section{CONCLUSION}

Gating errors cause a spurious reduction in SPECT assays of dyssynchrony magnitude. In our standard imaging practice, gating errors were common, and post hoc correction appears to be feasible. All SPECT studies for dyssynchrony assessment should be scrutinized for gating error and corrected when it is present.

\section{DISCLOSURE STATEMENT}

The costs of publication of this article were defrayed in part by the payment of page charges. Therefore, and solely to indicate this fact, this article is hereby marked "advertisement" in accordance with 18 USC section 1734.

\section{ACKNOWLEDGMENTS}

We are indebted to Ernest Garcia for his insights and encouragement. No potential conflict of interest relevant to this article was reported.

\section{REFERENCES}

1. Reuter S, Garrigue S, Barold SS, et al. Comparison of characteristics in responders versus nonresponders with biventricular pacing for drug-resistant congestive heart failure. Am J Cardiol. 2002;89:346-350.

2. Friehling M, Chen J, Saba S, et al. A prospective pilot study to evaluate the relationship between acute change in left ventricular synchrony after cardiac resynchronization therapy and patient outcome using a single-injection gated SPECT protocol. Circ Cardiovasc Imaging. 2011;4:532-539.

3. Boogers MM, Van Kriekinge SD, Henneman MM, et al. Quantitative gated SPECT-derived phase analysis on gated myocardial perfusion SPECT detects left ventricular dyssynchrony and predicts response to cardiac resynchronization therapy. J Nucl Med. 2009;50:718-725.

4. Henneman MM, Chen J, Dibbets-Schneider P, et al. Can LV dyssynchrony as assessed with phase analysis on gated myocardial perfusion SPECT predict response to CRT? J Nucl Med. 2007;48:1104-1111.

5. Chen J, Garcia EV, Folks RD, et al. Onset of left ventricular mechanical contraction as determined by phase analysis of ECG-gated myocardial perfusion SPECT imaging: development of a diagnostic tool for assessment of cardiac mechanical dyssynchrony. J Nucl Cardiol. 2005;12:687-695.

6. Galt JR, Garcia EV, Robbins WL. Effects of myocardial wall thickness on SPECT quantification. IEEE Trans Med Imaging. 1990;9:144-150.

7. Nichols K, Dorbala S, DePuey EG, Yao SS, Sharma A, Rozanski A. Influence of arrhythmias on gated SPECT myocardial perfusion and function quantification. J Nucl Med. 1999;40:924-934.

8. Nichols K, Yao SS, Kamran M, Faber TL, Cooke CD, DePuey EG. Clinical impact of arrhythmias on gated SPECT cardiac myocardial perfusion and function assessment. J Nucl Cardiol. 2001;8:19-30.

9. Henneman MM, Chen J, Ypenburg C, et al. Phase analysis of gated myocardial perfusion single-photon emission computed tomography compared with tissue Doppler imaging for the assessment of left ventricular dyssynchrony. J Am Coll Cardiol. 2007;49:1708-1714.

10. Trimble MA, Borges-Neto S, Honeycutt EF, et al. Evaluation of mechanical dyssynchrony and myocardial perfusion using phase analysis of gated SPECT imaging in patients with left ventricular dysfunction. J Nucl Cardiol. 2008;15:663-670.

11. Trimble MA, Borges-Neto S, Smallheiser S, et al. Evaluation of left ventricular mechanical dyssynchrony as determined by phase analysis of ECG-gated SPECT myocardial perfusion imaging in patients with left ventricular dysfunction and conduction disturbances. J Nucl Cardiol. 2007;14:298-307.

12. Trimble MA, Velazquez EJ, Adams GL, et al. Repeatability and reproducibility of phase analysis of gated single-photon emission computed tomography myocardial perfusion imaging used to quantify cardiac dyssynchrony. Nucl Med Commun. 2008;29:374-381.

13. Boogers MJ, Chen J, van Bommel RJ, et al. Optimal left ventricular lead position assessed with phase analysis on gated myocardial perfusion SPECT. Eur J Nucl Med Mol Imaging. 2011;38:230-238.

14. van der Veen BJ, Al Younis I, Ajmone-Marsan N, et al. Ventricular dyssynchrony assessed by gated myocardial perfusion SPECT using a geometrical approach: a feasibility study. Eur J Nucl Med Mol Imaging. 2012;39:421-429.

15. Cullom SJ, Case JA, Bateman TM. Electrocardiographically gated myocardial perfusion SPECT: technical principles and quality control considerations. $\mathrm{J} \mathrm{Nucl}$ Cardiol. 1998;5:418-425. 